\title{
Acute effects of decaffeinated coffee and the major coffee components chlorogenic acid and trigonelline on incretin hormones
}

\author{
Margreet R Olthof ${ }^{1 *}$, Aimée E van Dijk ${ }^{1}$, Carolyn F Deacon², Robert J Heine ${ }^{3}$, Rob M van Dam ${ }^{4}$
}

\begin{abstract}
Coffee consumption is associated with a lower risk of type 2 diabetes. We tested the hypothesis that this is mediated by incretin hormones by measuring the acute effects of decaffeinated coffee and coffee components on GLP-1 and GIP concentrations. A randomized cross-over trial of the effects of $12 \mathrm{~g}$ decaffeinated coffee, $1 \mathrm{~g}$ chlorogenic acid, $500 \mathrm{mg}$ trigonelline, and placebo on total and intact GLP-1 and GIP concentrations during an oral glucose tolerance test took place in fifteen overweight men. No treatment significantly affected the overall GLP-1 or GIP secretion pattern following an OGTT relative to placebo. Decaffeinated coffee slightly increased total GLP-1 concentration 30 minutes after ingestion (before the OGTT) relative to placebo ( $2.7 \mathrm{pmol} / \mathrm{L}, \mathrm{p}=0.03$ ), but this change did not correspond with changes in glucose or insulin secretion. These findings do not support the hypothesis that coffee acutely improves glucose tolerance through effects on the secretion of incretin hormones. Chronic effects of coffee and its major components still need to be investigated.
\end{abstract}

\section{Findings}

In prospective cohort studies, higher coffee consumption has been associated with a lower risk of type 2 diabetes $[1,2]$. Results were similar for caffeinated and decaffeinated coffee, suggesting that components other than caffeine are responsible for this association. Coffee is a major source of the phenolic compound chlorogenic acid [3] and the vitamin B3 precursor trigonelline [4] which have been shown to reduce blood glucose concentrations in animal studies [5-7]. One of the putative mechanisms underlying the beneficial effects of coffee on glucose homeostasis could include a stimulatory effect on the incretin hormones glucagon-like peptide 1 (GLP-1) and glucose-dependent insulinotropic peptide (GIP). Coffee components may affect GLP-1 secretion by delaying glucose absorption, resulting in a higher exposure of the distal ileum to glucose, and therefore stimulation of the intestinal $\mathrm{K}$ cells [8]. One study found that decaffeinated coffee increased GLP-1 concentrations [9] and two studies found that both decaffeinated coffee and caffeinated coffee intake decreased GIP

\footnotetext{
* Correspondence: margreet.olthof@falw.vu.nl

'Department of Health Sciences and the EMGO Institute for Health and Care Research, VU University Amsterdam, the Netherlands

Full list of author information is available at the end of the article
}

concentrations $[9,10]$. These results suggest that coffee may exert a glucose-lowering response mediated by stimulating incretin hormones. Data on the effects of chlorogenic acid and trigonelline on incretin concentrations are lacking. We therefore investigated the acute effects of decaffeinated coffee and the coffee components chlorogenic acid and trigonelline on total and intact GLP-1 and GIP concentrations in humans.

Fifteen male, healthy, non-smoking, overweight (body mass index $25.0-35.0 \mathrm{~kg} / \mathrm{m}^{2}$ ) coffee consumers were enrolled. All subjects provided written informed consent. The study design has been described previously [11]. Briefly, four supplements were tested in this crossover trial: 12 grams of decaffeinated instant coffee granules (Nescafé Gold, Nestlé, The Netherlands); 1 g chlorogenic acid; $500 \mathrm{mg}$ trigonelline; and $1 \mathrm{~g}$ mannitol as placebo. All supplements were dissolved in $270 \mathrm{~mL}$ water.

The study consisted of four visits separated by at least six days. During each visit participants ingested one of the supplements 30 minutes before a $75 \mathrm{~g}$ oral glucose tolerance test (OGTT). Seven venous blood samples were taken via a cannula in the antecubital vein on each visit following an overnight fast. The first two blood samples were taken 30 minutes and immediately before 
the start of the OGTT. The other 5 blood samples were taken 15, 30, 60, 90, and 120 minutes thereafter. For measurement of intact GLP-1 the DPP-4 inhibitor diprotin A ( $0.1 \mathrm{mmol} / \mathrm{l}$, final concentration) was added immediately after collection.

Plasma concentrations of total GLP-1, intact GLP-1 and total GIP were determined as previously described $[12,13]$. Intra- and inter assay coefficients of variation were $2 \%$ and $5 \%$, respectively for intact GLP- 1 and $<6 \%$ and $<15 \%$, respectively for both total GLP-1 and GIP. Results for glucose and insulin concentrations have previously been presented [11].

The area under the curve (AUC) values for GLP-1 and GIP were calculated using the trapezoidal method. Main treatment effects relative to placebo for AUC values and mean GLP-1 and GIP concentrations for individual time points were analyzed using linear mixed regression models. Bonferroni correction was used to adjust for multiple comparisons. All tests were two-sided and P values $<0.05$ were considered statistically significant. Analyses were conducted using SPSS 17.0.

None of the treatments affected the area under the curve values for total GLP-1, intact GLP-1, or total GIP during the OGTT (Table 1). Decaffeinated coffee slightly but significantly increased total GLP-1 concentration relative to placebo at 30 minutes after ingestion (before the OGTT) $(2.7 \mathrm{pmol} / \mathrm{L}, 95 \% \mathrm{CI}: 0.2 ; 5.3, \mathrm{p}=0.03)$. Chlorogenic acid and trigonelline did not significantly affect incretin concentrations relative to placebo at any of the time points (Figure 1).

In this randomized cross-over trial in healthy overweight men, decaffeinated coffee, chlorogenic acid and trigonelline did not significantly affect the overall response of GLP-1 or GIP to an OGTT relative to placebo. This is in line with the finding that these supplements did not affect glucose and insulin area under the curves in this study [11]. Decaffeinated coffee slightly increased the total GLP-1 concentrations at $30 \mathrm{~min}$ after ingestion (before the OGTT) relative to placebo. This

\begin{tabular}{|c|c|c|c|}
\hline & $\begin{array}{l}\text { TOTAL } \\
\text { GLP-1 }\end{array}$ & $\begin{array}{c}\text { INTACT GLP-1* } \\
(\text { pmol/L } \times 120 \mathrm{~min})\end{array}$ & GIP \\
\hline Placebo & $1720 \pm 180$ & $83 \pm 33$ & $6984 \pm 566^{* *}$ \\
\hline Chlorogenic acid & $1549 \pm 178$ & $140 \pm 47$ & $6656 \pm 620$ \\
\hline Decaffeinated coffee & $1809 \pm 152$ & $66 \pm 21$ & $6725 \pm 516$ \\
\hline Trigonelline & $1730 \pm 160$ & $94 \pm 39$ & $7512 \pm 619$ \\
\hline
\end{tabular}

*Intact GLP-1 concentrations were very low and a substantial number was at the limit of detection and were reported as zero.

${ }^{* *} \mathrm{n}=14$ ( 1 missing value at time point $15 \mathrm{~min}$.). small change, although statistically significant, did not coincide with changes in glucose and insulin levels [11]. Therefore this is probably a chance finding.

The lack of an effect of decaffeinated coffee on GLP-1 in our study is not in line with results of Johnston et al. [9] who found an increased response. They also found that ingestion of caffeinated and decaffeinated coffee lowered GIP concentrations. This may be explained by the fact that Johnston et al. [9] gave $25 \mathrm{~g}$ glucose together with coffee, whereas we gave $75 \mathrm{~g}$ glucose 30 min after ingestion of the supplement. Potential differences in the type of coffee used in both studies probably do not explain the different results since the type of coffee and the concentration of chlorogenic acid were similar in the two studies [9,11]. Greenberg et al. [10] did not find an overall effect of decaffeinated coffee on GIP concentrations, which is in line with our results. However, they did find that decaffeinated coffee lowered GIP concentrations at 60 minutes following ingestion of the coffee (before the OGTT) relative to placebo and caffeine. In that study supplements were ingested $60 \mathrm{~min}$ before the OGTT. We do not have data on the GIP concentrations $60 \mathrm{~min}$ following ingestion of the supplements alone, because the OGTT was administered at $30 \mathrm{~min}$ after ingestion of the supplements.

In conclusion, acute ingestion of decaffeinated coffee and its major components chlorogenic acid and trigonelline did not affect GLP-1 and GIP responses during the oral glucose tolerance test. These findings do not support the hypothesis that the putative beneficial effect of coffee on the development of type 2 diabetes can be explained by improved GLP-1 and GIP responses to meals. Nevertheless, it cannot be excluded that habitual coffee consumption in contrast to acute administration may have an effect on incretin secretion.

\section{Acknowledgements}

We would like to thank the participants of the Coffee Study for their participation; and the Clinical Research Unit Internal Medicine of the VU University Medical Centre for use of the facilities.

The research was financially supported by the Dutch Diabetes Research Foundation (Stichting Diabetes Fonds Nederland, grant no. 2006.11.020). This study is registered at the Dutch Trial Register http://www.trialregister.nl under identifier NTR 1051.

\section{Author details}

'Department of Health Sciences and the EMGO Institute for Health and Care Research, VU University Amsterdam, the Netherlands. ${ }^{2}$ Department of Biomedical Sciences, Panum Institute, University of Copenhagen, Denmark. ${ }^{3}$ Diabetes Center, Department of Endocrinology, VU University Medical Center, Amsterdam, the Netherlands. ${ }^{4}$ Department of Epidemiology and Public Health and Department of Medicine, Yong Loo Lin School of Medicine, National University of Singapore, Singapore.

Authors' contributions

All authors read and approved the final manuscript. $\mathrm{MO}, \mathrm{RH}$, and RvD conceived the study and obtained funding. MO and AvD coordinated the data collection and CD the laboratory measurements. MO conducted the data analysis and wrote the first draft of the manuscript. AvD, CD, RH \& RvD 
(A) Total GLP-1

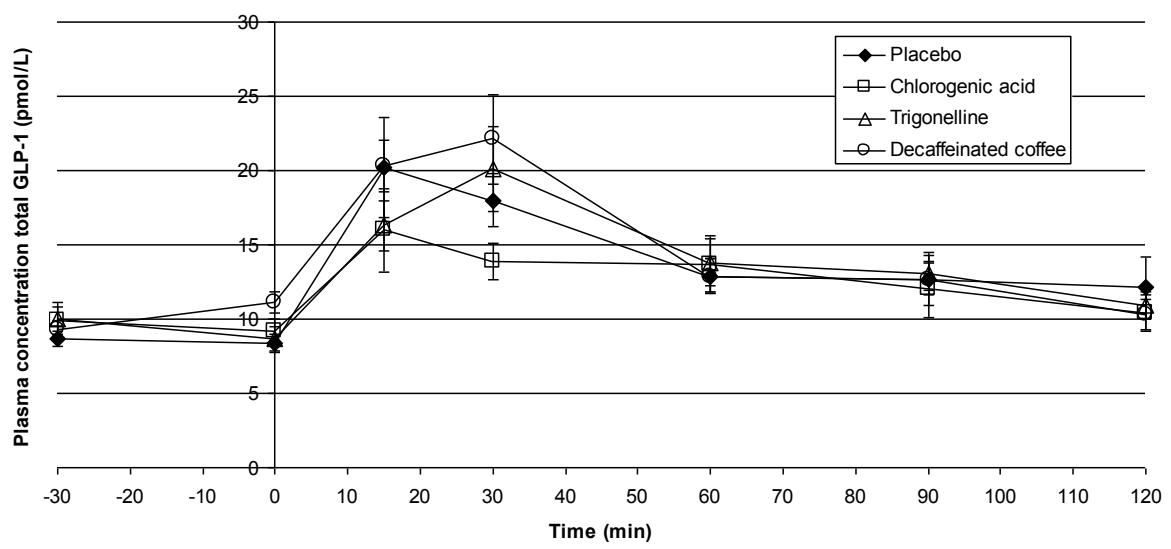

(B) Intact GLP-1

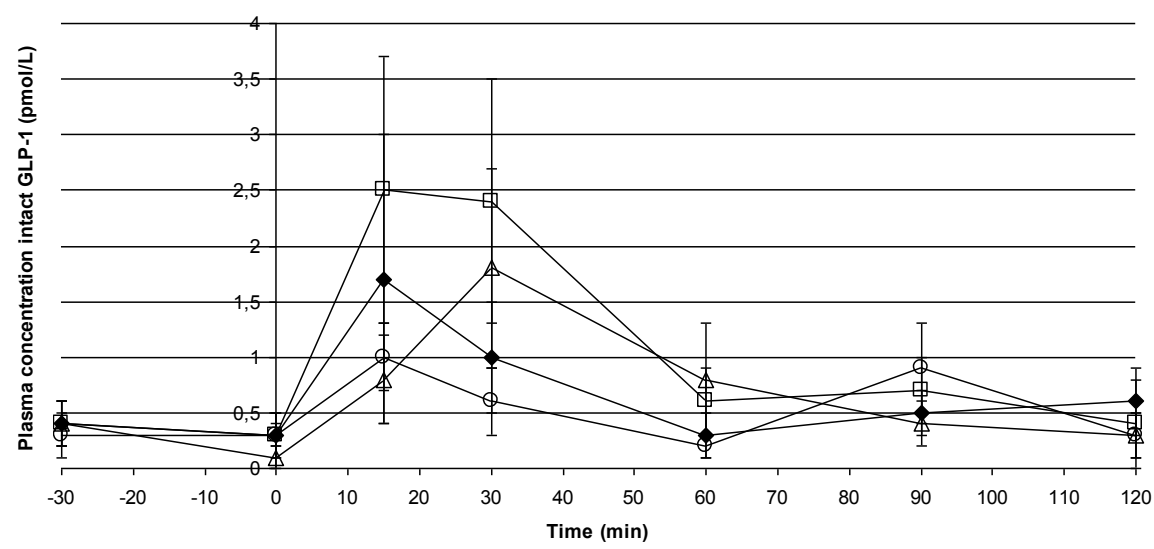

(C) GIP

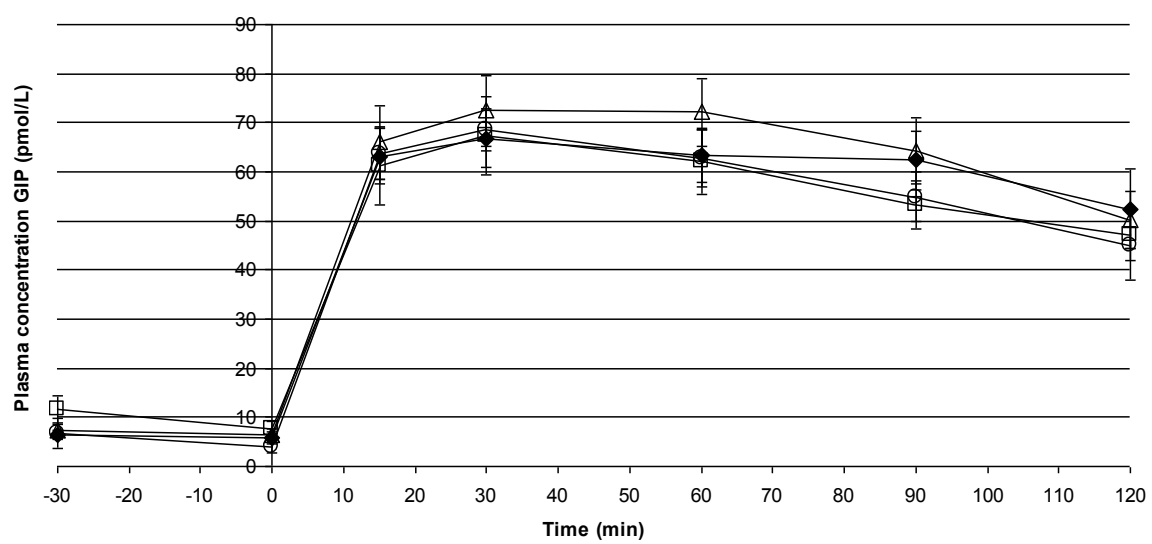

Figure 1 Mean ( \pm SE) plasma concentrations of total GLP-1 (A), intact GLP-1 (B) and GIP (C) before and throughout an OGTT, following ingestion of chlorogenic acid, decaffeinated coffee, trigonelline and placebo in $\mathbf{1 5}$ healthy overweight men. Figure $(A)$ : At $t=$ 0 decaffeinated coffee increased total GLP-1 concentration relative to placebo $(p=0.03)$. 


\section{Competing interests}

MRO, AEvD, CFD, RJH and RMvD have no relevant conflict of interest to

disclose. RJH is currently employed at Eli Lilly and Company, Indianapolis, IN.

Received: 5 October 2010 Accepted: 7 February 2011

Published: 7 February 2011

\section{References}

1. van Dam RM, Hu FB: Coffee consumption and risk of type 2 diabetes: a systematic review. JAMA 2005, 294:97-104.

2. van Dam RM, Willett WC, Manson JE, Hu FB: Coffee, caffeine, and risk of type 2 diabetes: a prospective cohort study in younger and middleaged U.S. women. Diabetes Care 2006, 29:398-403.

3. Clifford MN: chlorogenic acids and other cinnamates - nature, occurence and dietary burden. J Sci Food Agric 1999, 79:10.

4. Minamisawa M, Yoshida S, Takai N: Determination of biologically active substances in roasted coffees using a diode-array HPLC system. Anal Sci 2004, 20(2):325-8.

5. Mishkinsky J, Joseph B, Sulman FG: Hypoglycaemic effect of trigonelline. Lancet 1967, 2:1311-2.

6. Bassoli BK, Cassolla P, Borba-Murad GR, Constantin J, SalgueiroPagadigorria CL, Bazotte RB, da Silva RS, de Souza HM: Chlorogenic acid reduces the plasma glucose peak in the oral glucose tolerance test: effects on hepatic glucose release and glycaemia. Cell Biochem Funct 2008, 26:320-8.

7. Rodriguez de Sotillo DV, Hadley M: Chlorogenic acid modifies plasma and liver concentrations of: cholesterol, triacylglycerol, and minerals in (fa/fa) Zucker rats. J Nutr Biochem 2002, 13:717-26.

8. MCCarty MF: A chlorogenic acid-induced increase in GLP-1 production may mediate the impact of heavy coffee consumption on diabetes risk. Med Hypotheses 2005, 64:848-53.

9. Johnston KL, Clifford MN, Morgan LM: Coffee acutely modifies gastrointestinal hormone secretion and glucose tolerance in humans: glycemic effects of chlorogenic acid and caffeine. Am J Clin Nutr 2003, 78:728-33

10. Greenberg JA, Owen DR, Geliebter A: Decaffeinated coffee and glucose metabolism in young men. Diabetes Care 2010, 33:278-80.

11. van Dijk AE, Olthof MR, Meeuse JC, Seebus E, Heine RJ, van Dam RM: Acute effects of decaffeinated coffee and the major coffee components chlorogenic acid and trigonelline on glucose tolerance. Diabetes Care 2009, 32:1023-5.

12. Vollmer $\mathrm{K}$, Holst JJ, Baller B, Ellrichmann M, Nauck MA, Schmidt WE, Meier JJ: Predictors of incretin concentrations in subjects with normal, impaired, and diabetic glucose tolerance. Diabetes 2008, 57:678-87.

13. Vilsboll T, Krarup T, Sonne J, Madsbad S, Volund A, Juul AG, Holst JJ: Incretin secretion in relation to meal size and body weight in healthy subjects and people with type 1 and type 2 diabetes mellitus. $J$ Clin Endocrinol Metab 2003, 88:2706-13.

\section{doi:10.1186/1743-7075-8-10}

Cite this article as: Olthof et al:: Acute effects of decaffeinated coffee and the major coffee components chlorogenic acid and trigonelline on incretin hormones. Nutrition \& Metabolism 2011 8:10.

\section{Submit your next manuscript to BioMed Central and take full advantage of:}

- Convenient online submission

- Thorough peer review

- No space constraints or color figure charges

- Immediate publication on acceptance

- Inclusion in PubMed, CAS, Scopus and Google Scholar

- Research which is freely available for redistribution

Submit your manuscript at www.biomedcentral.com/submit
Biomed Central 